\title{
Epipericardial fat necrosis: an uncommon self- limiting cause of pleuritic chest pain
}

\author{
Sana Haq MD, Mitchell P. Wilson MD, Donna Manca MD MCISc
}

Cite as: CMAJ 2019 December 16;191:E1378-81. doi: 10.1503/cmaj.190703

A 55-year-old woman with a history of hypertension, fibromuscular dysplasia and previous stroke presented to our family practice clinic with a 4-day history of nonexertional chest pain. It started as acute left-sided pleuritic chest pain that radiated into the neck with associated numbness of the left arm. The patient rated the pain up to $8 / 10$ in severity; it was worse on deep inspiration, and when she lay flat or bent forward. The patient had searched the Internet regarding her symptoms and self-diagnosed pericarditis. She took ibuprofen and her symptoms improved. When she presented to the clinic, she was free of pain. She had no history of nausea, diaphoresis, peripheral edema, cough, shortness of breath, presyncope or acidic taste in her mouth. She had had no recent upper respiratory tract infections or any precipitating factors. She had no recent immobilization or unilateral leg swelling. Her medications were ramipril $5 \mathrm{mg}$, hydrochlorothiazide $25 \mathrm{mg}$ and acetylsalicylic acid (ASA) $81 \mathrm{mg}$ (for fibromuscular dysplasia) daily. She was taking ibuprofen (dose unknown) sporadically for the chest pain, with her last dose of ibuprofen at noon on the day of presentation. Her father had experienced a myocardial infarction at age 40 years; her family history was otherwise unremarkable. She was a nonsmoker.

A cardiovascular, respiratory and abdominal examination of the patient were all normal: she had normal first and second heart sounds, without any added heart sounds or murmurs. Her pulse was regular. There were no carotid bruits or swelling. She had equal air entry bilaterally, without any wheezes or crackles. Her abdomen was soft and non tender.

Given the atypical presentation of pericarditis, we began laboratory tests and basic investigations, including an electrocardiogram (ECG), quantitative D-dimer assay, troponin and a chest radiograph. We advised the patient to return to the emergency department on recurrence of pain and to continue on her current dose of ASA. Shortly after the patient's appointment, laboratory investigations showed a minimally elevated D dimer at 0.83 (nor$\mathrm{mal}<0.50) \mathrm{mg} / \mathrm{L}$ and the chest radiograph showed a small left pleural effusion with minor left basal atelectasis (Figure 1). Based on the patient's history and these results, we sent her to the emergency department for further assessment.

In the emergency department, the patient's vitals remained stable throughout her assessment. Cardiac and respiratory examinations were noncontributory. Serial troponins and ECG

\section{KEY POINTS}

- Epipericardial fat necrosis is an uncommon, benign cause of acute chest pain, typically presenting in healthy individuals without associated symptoms.

- The condition often presents as acute pleuritic chest pain, usually left sided, resembling other serious conditions such as myocardial infarction, pulmonary embolism and pneumonia.

- The diagnosis can be confirmed with computed tomography after other life-threatening conditions have been excluded.

- Conservative management with nonsteroidal anti-inflammatory medications and follow-up is often sufficient.

remained negative for acute myocardial infarction (Figure 2). A subsequent computed tomography (CT) pulmonary angiogram was negative for pulmonary embolism, but showed encapsulation of the epipericardial fat with internal and adjacent fat stranding consistent with a diagnosis of epipericardial fat necrosis (Figure 3).

After consultation with the respirology department, the patient received conservative management with acetaminophen and 1-week follow-up in clinic. By the time of her follow-up, her symptoms had resolved and a repeat chest radiograph 3 weeks after the onset of her initial symptoms showed a reduction of the left basal atelectasis with a smaller effusion. A repeat radiograph 3 months later was normal. At 6 months' follow-up, the patient had remained symptom free without recurrence of her chest pain.

\section{Discussion}

Epipericardial fat necrosis is an uncommon self-limiting condition that tends to occur in otherwise healthy individuals. The exact prevalence is unknown, ${ }^{1}$ and with the limited data available, there is no conclusive association of gender or other risk factors with this condition. ${ }^{2}$ The diagnosis remains an underrecognized and misdiagnosed condition; a recent retrospective study of patients presenting to the emergency department with chest pain and subsequent CT found the frequency of epipericardial fat necrosis to be $2 \% .^{3}$ Clinically, most cases have been described in the emergency department or inpatient setting. This case shows a presentation in the general practice setting 


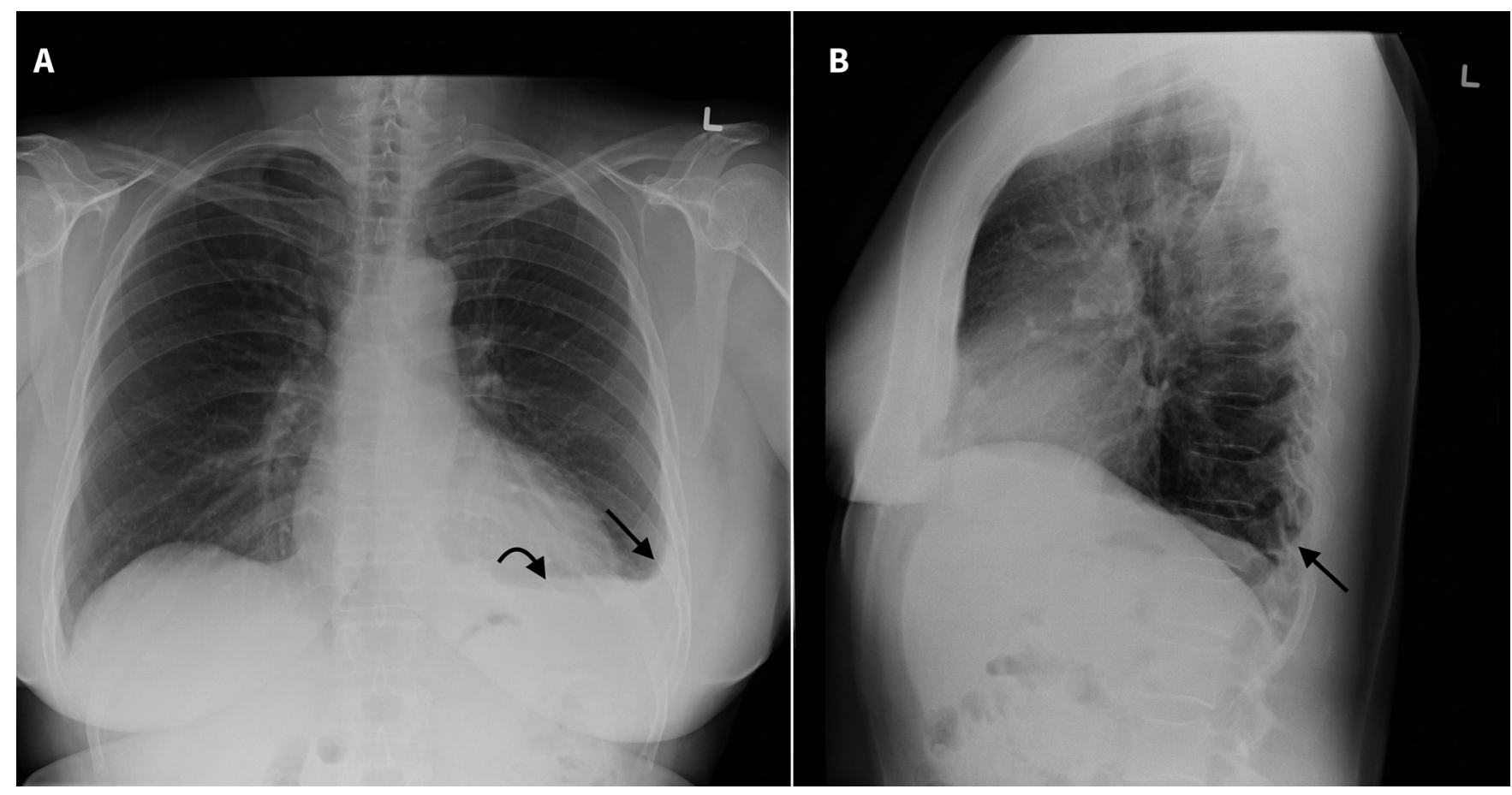

Figure 1: Frontal (A) and lateral (B) chest radiographs of a 55-year-old woman with pleuritic chest pain, showing a small left pleural effusion (arrows) with minor left basilar atelectasis (curved arrow).

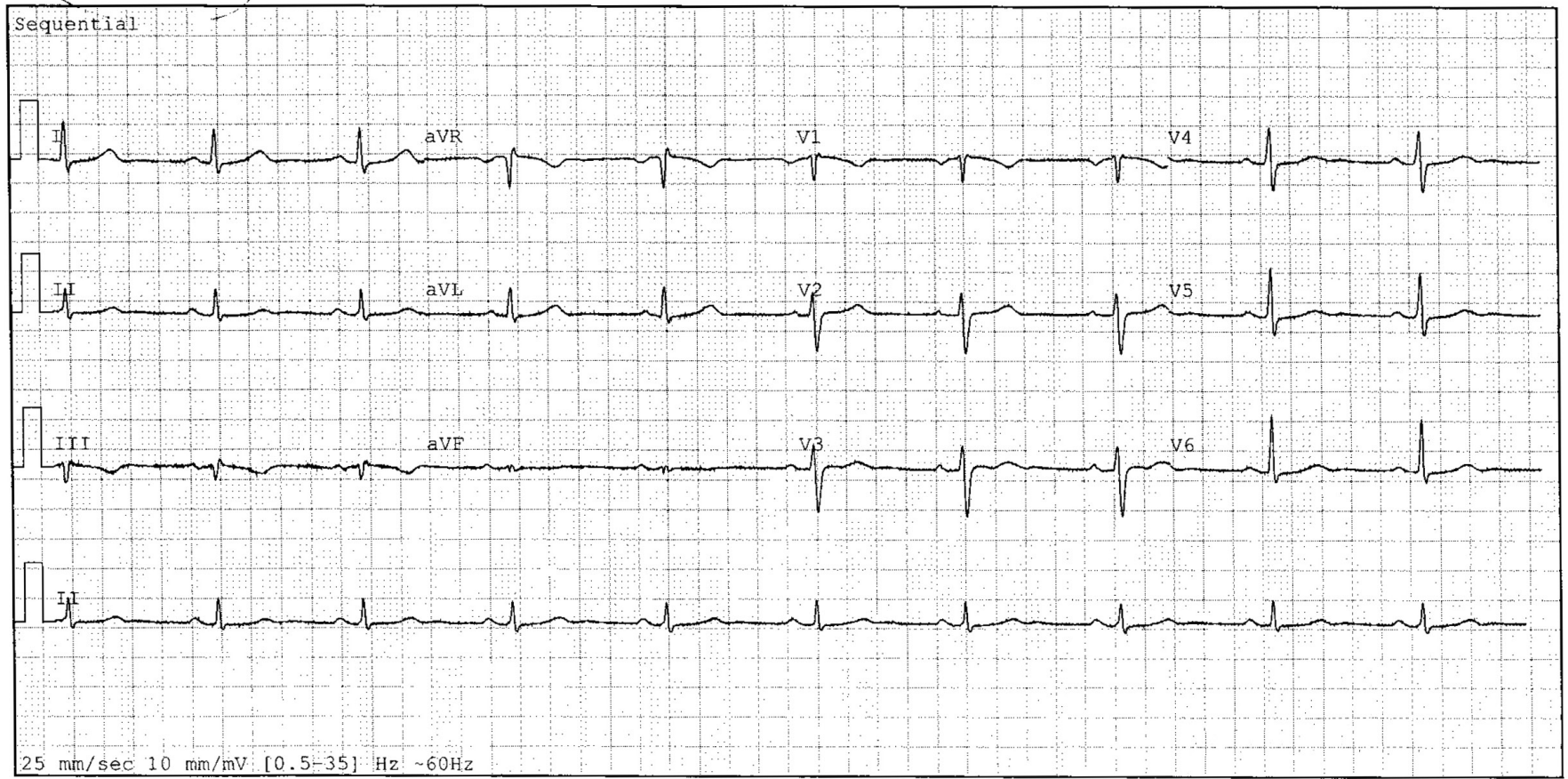

Figure 2: Electrocardiogram showing minor inferior repolarization disturbance and nonspecific changes.

and aims to increase awareness of this underdiagnosed mimic of potentially life-threatening conditions. The frequency of diagnosis has been shown to increase with improved awareness by radiologists. ${ }^{4}$

The pathogenesis of epipericardial fat necrosis remains poorly elucidated. Proposed mechanisms have included torsion of a pedicle-like lesion, resulting in ischemia; and increased capillary pressures during a Valsalva manoeuvre, resulting in hemorrhage into the epipericardial fat. ${ }^{3,5}$ The necrosis is centred within the epipericardium rather than the pericardium, implying that one of the terms previously used to describe this condition, "pericardial necrosis," is a misnomer. ${ }^{5}$ 


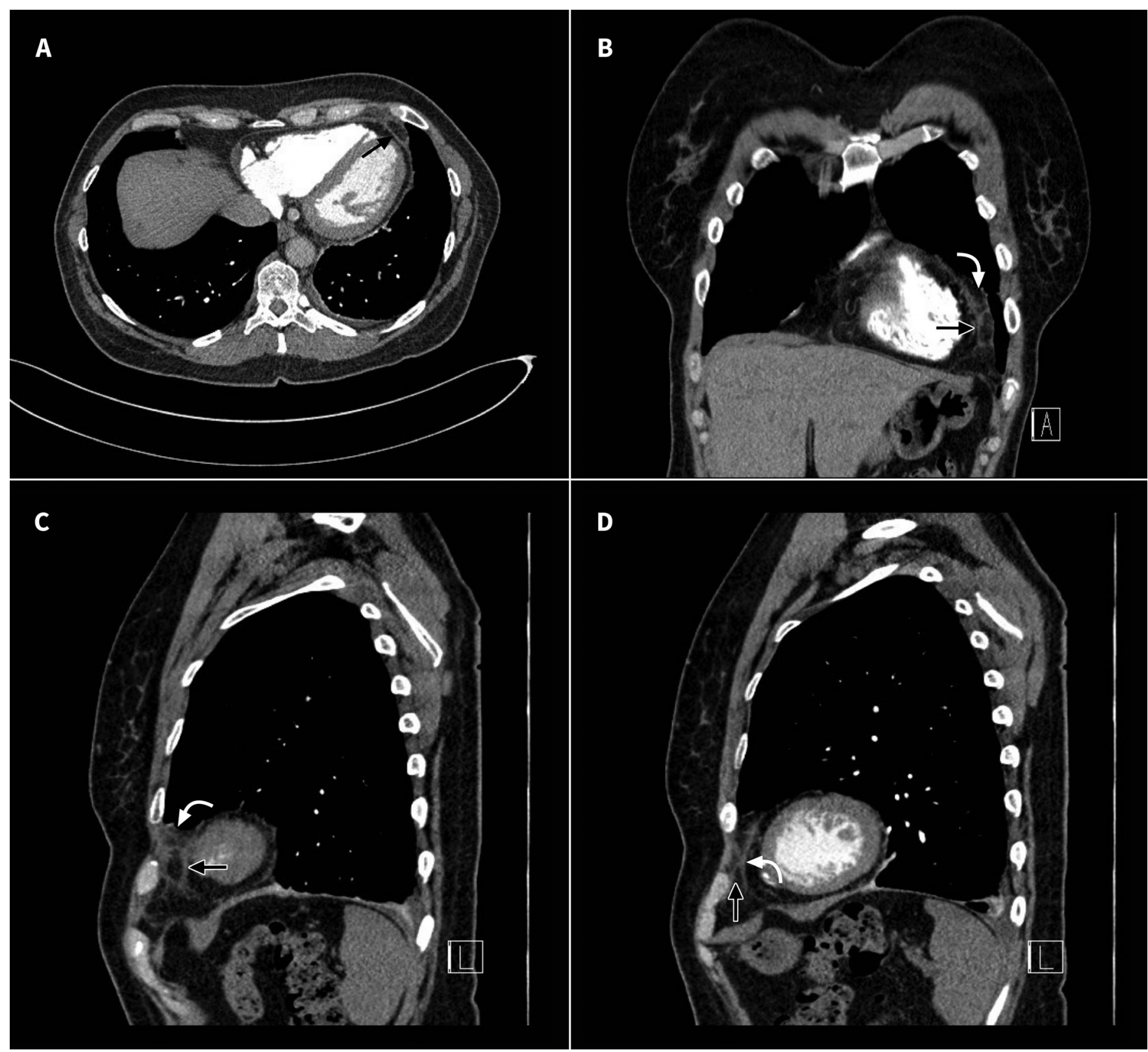

Figure 3: (A) Axial, (B) coronal and (C) sagittal contrast-enhanced computed tomography (CT) images of the chest show an ovoid encapsulated epipericardial fatty lesion (arrow) with surrounding epipericardial fat stranding (curved arrow) consistent with epipericardial fat necrosis. A sagittal CT (D) performed medial to the focus of fat necrosis shows regional reactive inflammation including epipericardial fat stranding (arrow), focal thickening of the adjacent pericardium (curved arrow), and a small reactive pleural effusion.

The condition often presents as acute pleuritic chest pain, resembling other serious etiologies such as myocardial infarction, pulmonary embolism and pneumonia. ${ }^{6}$ Pain is usually left sided and pleuritic, as the epipericardial fat at the left cardiophrenic angle is most often involved. ${ }^{3}$ Radiation to the neck, shoulders and upper arm has been described. ${ }^{6}$ The pain is frequently self-limited to only a few days but can last up to several weeks. ${ }^{5}$ Often there is no history of fever, cough or other associated symptoms or medication use. ${ }^{1,3}$ Historically, obesity had been considered a risk factor for epipericardial fat necrosis, but studies to date have failed to show an association between the 2 entities. ${ }^{3,5,6}$
The patient may demonstrate diaphoresis, tachycardia or tachypnea; otherwise, the clinical examination is often noncontributory, with negative findings on cardiopulmonary and abdominal assessment. ${ }^{1,7}$ Tenderness to palpation over the left anterior chest may be present. ${ }^{6}$ The presence of a friction rub on auscultation has been described in some patients with a coexisting pericarditis., ${ }^{6,7}$

Bloodwork and initial imaging are often noncontributory. ${ }^{5}$ Troponins should remain negative. Echocardiography often shows nonspecific or normal findings. ${ }^{1}$ A normal ECG is an important negative finding that could help differentiate necrosis of the epipericardium from inflammation of the pericardium. ${ }^{7}$ 
Mild elevation in inflammatory markers may be present, as was shown in our case. ${ }^{7}$

Initial chest radiography may be normal. If inflammation in the epipericardial fat is advanced, a circumscribed density can be seen within the fat at the cardiophrenic angle, although this is nonspecific. ${ }^{5}$ A small reactive pleural effusion on the side of involved fat may also be present. ${ }^{3}$

A chest CT is often the confirmatory test, demonstrating an ovoid encapsulated epipericardial fatty lesion with internal fat stranding. Regional inflammatory changes may be present, including stranding within the adjacent epipericardial fat, thickening of the adjacent pericardium and a small reactive pleural effusion. ${ }^{3,5,6}$ These secondary features were well demonstrated in the case of our patient. Similar fat stranding has also been reported with fat necrosis occurring in omental torsion and epiploic appendagitis, making it difficult to distinguish the lesions from a more serious process in the absence of awareness of this benign condition. ${ }^{7}$

Since 1957, cases of epipericardial fat necrosis have primarily been managed surgically. ${ }^{8}$ As awareness that the condition is usually benign increased in the early 21 st century, nonsurgical management of cases has been described. ${ }^{5,8}$ After more insidious pathologies have been excluded and epipericardial fat necrosis has been confirmed as the diagnosis, conservative management is preferred. Nonsteroidal anti-inflammatory medications and follow-up are often sufficient, with symptoms typically resolving within a few days. ${ }^{5,7,8}$ Follow-up CT studies show involution of the findings over the course of weeks to months. ${ }^{3,8}$ In rare cases, where the definitive CT appearance remains uncertain, more advanced imaging modalities such as magnetic resonance imaging, and even exploratory thoracotomy, have been used to exclude underlying malignancy. ${ }^{6}$ The need for further investigations is case dependent when the diagnosis remains uncertain, or an alternative, more serious condition is suspected.

\section{Conclusion}

Epipericardial fat necrosis is an uncommon but pertinent differential consideration for patients presenting with acute pleuritic chest pain and negative or near-normal laboratory tests. The combination of acute pleuritic chest pain accompanied by typical CT chest findings can help establish the self-limiting diagnosis with confidence. ${ }^{3,5}$

\section{References}

1. Giassi KS, Costa AN, Bachion GH, et al. Epipericardial fat necrosis: an underdiagnosed condition. Br J Radiol 2014;87:20140118.

2. Friedman YE, Gayer G, Livne Margolin M, et al. Epipericardial fat necrosis. Isr Med Assoc J 2018;5:327-8.

3. Giassi KS, Costa AN, Bachion GH, et al. Epipericardial fat necrosis: Who should be a candidate? AJR Am J Roentgenol 2016;207:773-7.

4. Giassi KS, Costa AN, Kairalla RA, et al. Epipericardial fat necrosis: increasing the rate of diagnosis by disseminating knowledge within a single institution. Radiol Bras 2018;51:62-3.

5. Pineda V, Cáceres J, Andreu J, et al. Epipericardial fat necrosis: radiologic diagnosis and follow-up. AJR Am J Roentgenol 2005;185:1234-6.

6. Fred HL. Pericardial fat necrosis: a review and update. Tex Heart Inst J 2010;37:82-4.

7. Nguyen DN, Tran CD, Rudkin SM, et al. Epipericardial fat necrosis: uncommon cause of acute pleuritic chest pain. Radiol Case Rep 2018;13:1276-8.

8. Runge T, Greganti MA. Epipericardial fat necrosis - a rare cause of pleuritic chest pain: case report and review of the literature. Arch Med Sci 2011;7:337-41.

\section{Competing interests: None declared.}

This article has been peer reviewed.

The authors have obtained patient consent.

Affiliations: Departments of Family Medicine (Haq, Manca), and Radiology and Diagnostic Imaging (Wilson), University of Alberta, Edmonton, Alta.

Contributors: All authors contributed substantially to the conception and design of the work. Mitchell Wilson and Donna Manca contributed imaging. All authors drafted the manuscript, revised it critically for important intellectual content, gave final approval of the version to be published and agreed to be accountable for all aspects of the work.

Correspondence to: Sana Haq, shaq@ualberta.ca 